\title{
Elementos clave para la introducción de la perspectiva de género en las infraestructuras ciclistas
}

\author{
Key elements for the introduction of the gender approach on \\ cycling infrastructures
}

\author{
Anna Obach Lapieza ${ }^{1}$ y María Ramos Sanz \\ Fecha de recepción: 06-06-2020 - Fecha de aceptación: 04-08-2020 \\ Hábitat y Sociedad (ISSN 2173-125X), n. ${ }^{\circ}$ 13, noviembre de 2020, pp. 87-105. \\ http://dx.doi.org/10.12795/HabitatySociedad.2020.i13.06
}

\section{Summary}

Gender mainstreaming generated a wide diversity of innovative measures and interventions in new areas not explored until the moment. This is the case of mobility policies, linked to the sustainable urban design and the measures for the environment's improvement.

The literature review and the analysis of the cities' experiences, allow us to establish some criteria to audit the level of inclusivity of transport infrastructures. In the case of cycling infrastructures, the following criteria have to be taken into account: security, ensuring road safety elements and adequate lighting; conciliation, connecting areas of the city linked to child care, elderly care, daily shopping and personal dealings, through Geographic Information System (GIS) analysis; apply an inclusive and participatory approach, from the comprehension that there is a diverse citizenship with multiple needs and that these have to be taken into account during the design of these policies; and the visibility of cyclist women, as a tool of empowerment and promotion of the use of the bicycle.

\section{Key words}

Gender; Transport; Sustainability; Bicycle and GIS

\section{Resumen}

La transversalidad de género ha producido una gran diversidad de medidas e intervenciones novedosas en ámbitos que hasta el momento no se habían explorado. Este es el caso de las políticas de movilidad, muy ligadas al diseño urbano sostenible y a las medidas por la mejora del medioambiente.

La revisión de literatura y el análisis de las experiencias que algunas ciudades están desarrollando permiten establecer criterios para auditar en qué niveles las infraestructuras de transporte son inclusivas. En el caso de las infraestructuras ciclistas es necesario tener en cuenta: la seguridad, garantizando elementos de seguridad vial y una iluminación adecuada; la conciliación, conectando zonas de la ciudad vinculadas a los cuidados de infantes, de personas mayores, las compras cotidianas y las gestiones personales, mediante el análisis por Sistema de Información Geográfica (SIG); la aplicación de una mirada integradora y participativa, desde la comprensión que existe una ciudadanía diversa con múltiples necesidades y estas deben ser tenidas en cuenta durante el diseño de estas políticas; y la visibilidad de las mujeres ciclistas, como herramienta de empoderamiento y promoción del uso de la bicicleta.

\section{Palabras clave}

Género; Transporte; Sostenibilidad; Bicicleta y SIG

1 BAa Consultors S.L. E-mail: aobach@baaconsultors.com. ORCID: 0000-0002-7595-6790
2 E-mail: arestaingenieria@gmail.com. ORCID: 0000-0001-5400-390X 


\section{Introducción}

Con la incorporación de la perspectiva de género en el diseño de las políticas públicas, las ciudades se enfrentan a diversos retos. La introducción de conceptos y metodologías que permitan establecer espacios sin discriminación para el conjunto de la ciudadanía requiere de la comprensión de elementos teóricos, así como del aprendizaje de experiencias de otras ciudades que sirvan como referentes.

Se ha escrito sobre la incorporación de la perspectiva de género en diferentes ámbitos (Alfama i Guillén, 2012) y existen referentes (Alonso Álvarez, 2015) que pueden establecer una base para que cada vez más ciudades se atrevan a iniciar estos procesos. A pesar de ello, no existen fórmulas o planes que puedan adaptarse a todas las situaciones y ciudades. Por tanto, la adopción de este tipo de estrategias son una ocasión para reflexionar y explorar las soluciones que mejor se adaptan a las necesidades y características de nuestras ciudades. Poniendo en cuestión las condiciones actuales de discriminación que se desarrollan en nuestra ciudad y ámbito de actuación.

La transversalidad de género, como corriente que busca hacer de la perspectiva de género un elemento central del diseño e implementación de las políticas públicas (Grupo de especialista en Mainstreaming, 1999), ha permitido que muchas ciudades hayan iniciado procesos en áreas sectoriales que hasta el momento no estaban exploradas. Tradicionalmente las áreas de políticas públicas que incorporan la perspectiva de género eran del tipo social, lideradas por los departamentos de igualdad, e incidiendo en áreas como cultura o juventud. La transversalidad de género conlleva ampliar la incidencia de la perspectiva de género en nuevas áreas de actuación. Dado que uno de los principales objetivos de la estrategia de transversalidad es aplicar esta perspectiva de forma transversal en cada una de las áreas de las que se compone el organismo. Así, el género pasa a ser un elemento a tener en cuenta en nuevas áreas como la economía, los deportes o la movilidad.

En las próximas páginas nos disponemos a explorar la introducción de la perspectiva de género en el diseño de las políticas de movilidad, con especial atención en las infraestructuras ciclistas. Analizaremos los elementos teóricos que nos permitirán afrontar el diseño de estas infraestructuras de forma integradora y no discriminatoria, reflexionando cómo diferentes elementos inciden en el menor o mayor uso de estas infraestructuras por parte de las mujeres. Estableceremos los elementos básicos que deben introducirse en las infraestructuras ciclistas para poder considerar que se ha tenido en cuenta la perspectiva de género en su diseño; aportando un check-list que sirva de herramienta de trabajo durante este proceso.

\section{Políticas públicas y género}

La introducción de la perspectiva de género en el diseño de las políticas públicas implica analizar cómo las políticas públicas responden a las necesidades de la ciudadanía, desde la comprensión que el género tiene un gran impacto en determinar estas necesidades. Y por tanto cuestionar la concepción de la ciudadanía como personas con necesidades homogéneas, incorporando las necesidades de mujeres y hombres en todas las fases del diseño de estas políticas. Bajo la premisa que las necesidades de las mujeres no estaban ya incorporadas con anterio- 
ridad en estas políticas, y por tanto generan situaciones de desigualdad estructural (EIGE, 2019).

Es necesario analizar el impacto que estas políticas tienen en la reproducción de roles de género tradicionales, como puede ser la segregación sexual del trabajo, el protagonismo de las mujeres en los roles de cuidadoras, o en situaciones de discriminación como es el acoso sexual. Una vez analizadas las necesidades a las que debemos dar respuesta, e identificados aquellos condicionantes que perpetúan situaciones de discriminación, será necesario rediseñar las políticas públicas y establecer las condiciones que respondan a estos elementos. Contribuyendo a reducir la perpetuación de estas desigualdades y discriminaciones por cuestión de género.

De este modo, las políticas públicas con perspectiva de género construyen escenarios donde los organismos públicos responden a las necesidades de la ciudadanía en esferas diversas de la vida cotidiana, más allá de la esfera productiva o el ámbito laboral. Incorporando necesidades de las tareas de cuidado y del ámbito comunitario, ofreciendo servicios vinculados a estas esferas y ajustando los servicios existentes a estas actividades. Así como teniendo en cuenta otros condicionantes que se desarrollan bajo el marco de la discriminación de género, como puede ser el acoso sexual.

Bajo este marco conceptual se comprende que la perspectiva de género es un elemento a tener en cuenta durante todo el ciclo de política pública, compuesto por las fases: identificación de la problemática o diagnóstico, diseño de la política pública, implementación y evaluación.

\section{Movilidad y género}

El urbanismo feminista defiende que la movilidad no es neutral en términos de género (Col-lectiu Punt 6, 2019). Mujeres y hombres tienen experiencias diversas en su uso del transporte, así como expresan diferentes necesidades y tipologías de uso de los modos de transporte. La movilidad con perspectiva de género tiene en cuenta las diferencias que muestran mujeres y hombres en los motivos de desplazamiento, los patrones de estos desplazamientos, y sus experiencias en el uso de los diferentes modos de transporte. Mostrando las carencias de las infraestructuras de transporte de nuestras ciudades, derivadas de la concepción androcéntrica a partir de la cual fueron diseñadas (Miralles-Guasch, 2010).

Encontramos que las políticas públicas que tienen mayor incidencia en las cuestiones de movilidad acostumbran a ser las políticas locales. Por supuesto, el contexto regional o estatal tendrá su influencia, pero el abordaje de una movilidad con perspectiva de género tiene un protagonismo de ciudades.

Como primer paso, intentaremos comprender las diferencias de género en cuanto a los motivos de desplazamiento, patrones y experiencias, que nos permitirán determinar qué elementos debemos tener en cuenta cuando buscamos incrementar el uso de la bicicleta por parte de las mujeres, dándonos información de los condicionantes que determinan este uso.

\section{Motivos de desplazamiento}

Diversos estudios han demostrado cómo mujeres y hombres se desplazan por motivos diferenciados (Beall, 1996; Campos de Michelena, 
Cuadro 1. Motivo del desplazamiento según género. Fuente: Elaboración propia a partir de los datos de la Encuesta de movilidad en día laborable (ATM, 2018).
Cuadro 2. Modo de transporte agregado según género. Fuente: Elaboración propia a partir de los datos de la Encuesta de movilidad en día laborable (ATM, 2018).
1996; Bofill, 2005; Ciocoletto, 2014; por citar algunos ejemplos). La división sexual del trabajo y el desempeño de las tareas de cuidado protagonizado por mujeres es uno de los elementos que explica esta diferencia. Según datos de la Encuesta de Movilidad en día laborable de 2018 (ATM, 2018) realizada en la provincia de Barcelona, se observan diferencias de género en los motivos de los desplazamientos por los que mujeres y hombres se desplazan de forma cotidiana.

En el Cuadro 1 podemos observar cómo los hombres se desplazan en mayores ocasiones por motivos de movilidad ocupacional, es decir, por motivos vinculados a estudios, trabajo o gestiones del trabajo; y las mujeres se desplazan más frecuentemente por motivos de movilidad personal, como las compras cotidianas y no cotidianas, ir al médico o al hospital, visitar a amistades y familiares, acompañar y cuidar a otras personas, gestiones personales, ocio, para pasear u otros motivos.

\begin{tabular}{|l|c|c|c|}
\hline Motivo del desplazamiento & Mujeres & Hombres & Total \\
\hline $\begin{array}{l}\text { Movilidad ocupacional (estudios o tra- } \\
\text { bajo) }\end{array}$ & $17,2 \%$ & $22,1 \%$ & $19,6 \%$ \\
\hline $\begin{array}{l}\text { Movilidad personal (compras, ges- } \\
\text { tiones personales, ocio, acompañar a } \\
\text { otras personas...) }\end{array}$ & $40,8 \%$ & $35,1 \%$ & $38,0 \%$ \\
\hline Vuelta a casa o al domicilio & $42,0 \%$ & $42,8 \%$ & $42,4 \%$ \\
\hline
\end{tabular}

Por tanto, las mujeres requieren desplazarse con mayor frecuencia por motivos vinculados con las tareas de cuidado, a menudo acompañando a otras personas. Mientras que los hombres se desplazan por motivos laborales, muy a menudo en solitario. El motivo del desplazamiento determinará si las personas van acompañadas de otras personas durante el desplazamiento, pero también puede determinar los patrones de desplazamiento, como veremos en el siguiente apartado.

\section{Patrones de desplazamiento}

Los estudios también demuestran que mujeres y hombres utilizan diferentes modos de transporte. El Cuadro 2 nos ofrece datos sobre el modo de transporte que utilizan mujeres y hombres en sus desplazamientos cotidianos (ATM, 2018). Las mujeres utilizan en mayor medida que los hombres los modos de transporte activos (caminando, bicicleta y vehículos de movilidad personal) y el transporte público (autobús, metro, otros ferroviarios y el resto de la red de transporte público), mientras que los hombres predominan entre la ciudadanía que utiliza vehículos privados (coches, motos, furgonetas, camiones u otros vehículos privados).

\begin{tabular}{|l|c|c|c|}
\hline Modo de transporte & Mujeres & Hombres & Total \\
\hline Movilidad activa & $47,0 \%$ & $40,8 \%$ & $43,9 \%$ \\
\hline Transporte público & $21,1 \%$ & $14,6 \%$ & $17,9 \%$ \\
\hline Vehículo privado & $31,9 \%$ & $44,7 \%$ & $38,1 \%$ \\
\hline
\end{tabular}

Además de poder caracterizar los patrones de desplazamiento de mujeres y hombres por sus motivos y modos de transporte, existen 
otros elementos estrechamente vinculados a estas características. Elementos que establecen diferencias en los patrones de desplazamiento generales de mujeres y hombres.

Se ha observado que las mujeres acostumbran a realizar distancias más cortas que los hombres, debido a gestiones personales o familiares (Judge, 2011), con desplazamientos a lugares más cercanos de la casa, encadenando viajes y diferentes modos de transporte, con desplazamientos repartidos durante el día, fuera de horas punta y con reticencias a desplazarse durante la noche (D-G for Research and Innovation, 2011; Col-lectiu Punt 6, 2019); mientras que los hombres realizan desplazamientos más uniformes, de tipo pendular, de la vivienda al trabajo (Zucchini, 2015), habitualmente durante las horas punta (ADB, 2013).
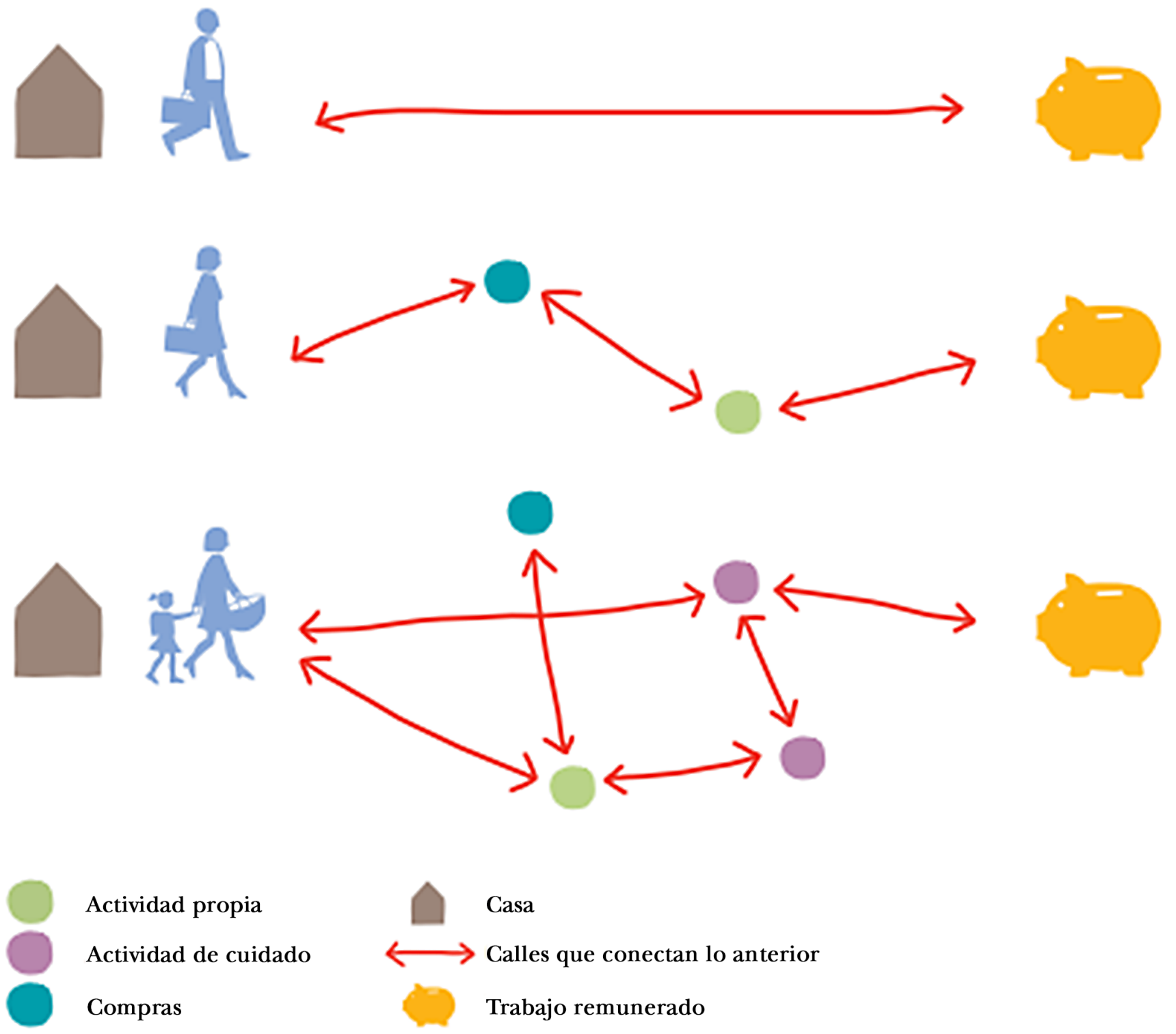

Actividad propia

Actividad de cuidado

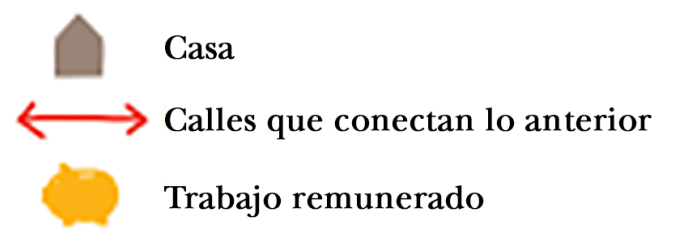

Compras

Figura 1. Itinerarios cotidianos, ruta de casa al trabajo. Fuente: Cioccoletto y Col.lectiu Punt 6, 2014. nes: desplazamientos pendulares en el caso de los hombres, y desplazamientos multimodales o intermedios en el caso de las mujeres, que se intensifican en el caso de ser mujeres al cuidado de personas dependientes (ya sean infantes o personas mayores). 
Estas diferencias entre los patrones de desplazamiento de mujeres y hombres están fuertemente vinculadas a:

- Roles de género en cuestiones de responsabilidad de las tareas de cuidados (motivo del desplazamiento, número de desplazamientos a lo largo del día y distancias cortas cerca del hogar).

- Una mayor presencia de la mujeres en los contratos a tiempo parcial (desplazamientos fuera de las horas punta).

\section{Experiencia en el transporte}

Además de los elementos que determinan los patrones de desplazamiento de mujeres y hombres, derivadas de las diferencias de género en cuanto a responsabilidad de los cuidados u otros elementos como la presencia en el mercado de trabajo, existen diferencias de género determinadas por cuestiones de responsabilidad ambiental y seguridad. Los estudios demuestran que las mujeres son más propensas a utilizar los modos de transporte más sostenibles por motivaciones medioambientales (CIVITAS, 2014), y que prefieren no desplazarse durante la noche por motivos de seguridad. Un ejemplo de esta falta de seguridad serían la falta de luz, que puede inducir miedo a asaltos físicos o sexuales, o los transportes públicos abarrotados, que pueden hacer incrementar el riesgo al acoso sexual (EBRD, 2011).

\section{Consecuencias del transporte en la vida cotidiana de las mujeres}

El modo en el que los sistemas e infraestructuras de transporte están habitualmente diseñados en nuestras ciudades, no concuerda con las necesidades, patrones y experiencias de las mujeres. Tradicionalmente, estos sistemas e infraestructuras han estado concebidas desde una perspectiva androcéntrica, con las necesidades y patrones de los hombres como únicas y universalizables a toda la ciudadanía (MirallesGuasch, 2010).

Esto implica que existe una centralidad de los modos de transporte mayormente usados por hombres, como es el coche. E implica que durante el diseño de sistemas de transporte no se tienen en cuenta los patrones de desplazamiento de las mujeres. O se tienen en cuenta de forma secundaria, y por tanto en muchas ocasiones se penalizan.

Por poner un ejemplo muy sencillo, pero muy ilustrativo, en el ámbito del transporte público. Habitualmente las tarifas y billetes de transporte se conciben siguiendo un modelo de movilidad pendular, en la que la personas usuaria toma el transporte público una o dos veces al día (ida y vuelta al trabajo) en un solo tramo (permitiendo el uso de un mismo billete para realizar cambios entre diferentes infraestructuras de transporte público, del metro al autobús, por ejemplo). De esta manera se penalizan los múltiples usos de un mismo transporte en cortos períodos de tiempo (por ejemplo, bajar del autobús de camino a casa para realizar una gestión y volver a subir a la misma línea de autobús para finalizar el trayecto hasta casa). Penalizando también a las personas que realizan diversos viajes cortos, utilizando múltiples modos de transporte en diferentes momentos del día, que como hemos visto son en su mayoría mujeres.

Además de los condicionantes en los modos de uso generales, existen por otro lado las condiciones de seguridad de estas infraestructuras 
de transporte. Estas condiciones de seguridad tienen un efecto en el uso que las mujeres harán de estas infraestructuras. Según la sensación de seguridad que ofrezca en términos de seguridad física directamente vinculada al uso de estas infraestructuras, por factores de aversión al riesgo más presentes en mujeres, pero también por motivos de seguridad en cuestiones de violencia, como son los asaltos o el acoso sexual.

Hasta este punto hemos analizado las diferencias de género que determinan los motivos de desplazamiento, patrones de desplazamiento y experiencia en el transporte de modo general. Estos elementos nos ayudan a comprender el marco teórico de la perspectiva de género en el ámbito del transporte.

A continuación veremos cómo influyen estos elementos al uso de la bicicleta, y analizaremos algunos elementos clave para poder diseñar infraestructuras ciclistas de un modo inclusivo y no discriminatorio.

\section{Bicicleta y género}

A pesar de que las mujeres representan un mayor porcentaje de la población que se desplaza en modos de movilidad activa, no son las que más utilizan todos los diferentes métodos de este tipo de movilidad. En el Cuadro 3 podemos observar cómo la mayor parte de las mujeres que se desplazan con modos de transporte activo lo realizan a pie, en un porcentaje mayor que los hombres (ATM, 2018). Mientras que los hombres usan en mayor medida que las mujeres modos de transporte activo como la bicicleta y otros vehículos de movilidad personal, como son las sillas de ruedas, los patinetes y los segways.

\begin{tabular}{|l|c|c|c|}
\hline Modo de transporte activo & Mujeres & Hombres & Total \\
\hline Caminando & $46,1 \%$ & $38,1 \%$ & $42,2 \%$ \\
\hline Bicicleta & $0,6 \%$ & $2,2 \%$ & $1,4 \%$ \\
\hline $\begin{array}{l}\text { Silla de ruedas u otros vehículos } \\
\text { de movilidad personal }\end{array}$ & $0,3 \%$ & $0,5 \%$ & $0,4 \%$ \\
\hline Total modo de transporte activo & $47,0 \%$ & $40,8 \%$ & $43,9 \%$ \\
\hline
\end{tabular}

Aunque los datos sobre otros vehículos de movilidad personal, como los patinetes y segways, son poco representativos y solo recientemente introducidos en este tipo de encuestas, parece haber indicios que estos modos de transporte presentan una tendencia de uso muy similar a las bicicletas.

Según datos del Barómetro de la Bicicleta en España (DGT, 2019) las principales razones para no utilizar la bicicleta es la preferencia por otros medios, razones de salud y sensación de peligrosidad por la presencia de mucho tráfico (motivo destacado en la ciudad de Barcelona). Lamentablemente, estos datos no estaban desagregados por género, hecho que nos impide observar si existe una razón diferenciada para no utilizar la bicicleta entre las mujeres y hombres encuestados.

Según el mismo informe, las mujeres que circulan en bicicleta declaran ir más por la acera que los hombres, y expresan más actitudes negativas de vehículos a motor (ATM, 2018). Así, con estos datos, parece posible afirmar que la sensación de inseguridad podría ser uno de los elementos que explica el menor porcentaje de mujeres que decide usar la bicicleta como medio de transporte para sus desplazamientos
Cuadro 3. Modos de transporte activo según género. Fuente: Elaboración propia a partir de los datos de la Encuesta de movilidad en día laborable (ATM, 20181 . 
cotidianos. O por lo menos cabe pensar que esta sensación de inseguridad es uno de los elementos que influye en la decisión de muchas mujeres en cuanto al uso de la bicicleta.

Como veremos en los apartados siguientes, existen otras razones por las cuales las mujeres utilizan otros medios de transporte frente a la bicicleta. Afrontar los elementos que presentaremos a continuación por parte de las ciudades durante el ciclo de sus políticas públicas y el diseño de sus infraestructuras de transporte, deberá de permitir a las mujeres escoger el método de transporte que mejor se adapte a sus necesidades o preferencias en igualdad de condiciones. Permitiendo también un uso más amable de los diferentes métodos de transporte activo y público por parte del conjunto de la ciudadanía.

\section{Ciudades amigables con el transporte activo}

A partir de la declaración de los objetivos de desarrollo sostenible de las Naciones Unidas, las ciudades han impulsado algunas iniciativas destinadas al cumplimento de estos objetivos. El Objetivo 11: Lograr que las ciudades sean más inclusivas, seguras, resilientes y sostenibles está estrechamente vinculado con las políticas de movilidad. Interpelando a los organismos encargados del diseño del entorno urbano y de infraestructuras de transporte a potenciar el uso de modos de transporte activo y el transporte público.

Parte de las acciones que las ciudades pueden tomar encaminadas a potenciar el uso del transporte activo pasan por potenciar este uso entre sectores de la población que presentan una tendencia a un menor uso de modos de transporte activo. En el caso de la temática que nos ocupa, podemos contemplar a las mujeres como un sector de la población con gran potencial de uso de la bicicleta que hace falta explorar.

Para potenciar el uso de la bicicleta por parte de las mujeres, deberemos tener en cuenta los aspectos vinculados con la seguridad, la conciliación, la participación y la visibilidad. En los siguientes apartados describiremos cómo estos elementos determinan el uso que realizan las mujeres de las infraestructuras ciclistas. Y aportaremos algunos datos sobres las condiciones mínimas para garantizar la inclusión de la perspectiva de género en su diseño.

Cabe destacar que potenciar el uso de la bicicleta entre las mujeres a partir de los elementos que vamos a mencionar, permite ampliar la población que utiliza este método de transporte. Reforzando los elementos de seguridad y participación, observaremos cómo el uso de la bicicleta aumenta también entre personas jóvenes, mayores o personas con discapacidad, por poner algunos ejemplos.

\section{Seguridad}

La tendencia apunta a que las mujeres tienen mayor aversión al riesgo que los hombres, y estos presentan actitudes temerarias en mayor medida que las mujeres. Así, si las infraestructuras ciclistas de nuestras ciudades son percibidas como infraestructuras inseguras, serán utilizadas mayormente por hombres. Especialmente aquellos más jóvenes y en mejor estado de salud. Según el estudio de Ainsley Henry Judge (2011), las mujeres prefieren los carriles bici segregados del tráfico mo- 
torizado, en paralelo, y a poder ser con separación física y con una distancia de seguridad.

Por tanto, para ampliar el uso de la bicicleta como medio de transporte en las ciudades, hace falta fortalecer estas infraestructuras en cuestiones de seguridad. Más adelante detallaremos los principales elementos del diseño de una infraestructura ciclista que deberemos tener en cuenta para garantizar su seguridad. Los principios que deben regir la incorporación de estos elementos de seguridad, según Col·lectiu Punt 6 (2011) en su informe Construyendo entornos seguros desde la perspectiva de género, son:

- Señalización: saber donde se está y adónde se va. Garantizando que existen suficientes señales para orientar a las personas usuarias durante todo el recorrido.

- Visibilidad: ver y ser visto/a.

- Concurrencia de personas: oír y ser oído/a.

- Vigilancia formal y acceso a la ayuda: poder escapar y obtener auxilio.

- Planificación y mantenimiento de los lugares: vivir en un ambiente limpio y acogedor.

- Participación de la comunidad: actuar en conjunto.

Con estos principios en mente podremos diseñar infraestructuras que aportan seguridad y sensación de seguridad al conjunto de la población, y especialmente a las mujeres. A continuación detallamos los elementos del diseño que se verán influenciados por estos principios:

- Separación del carril bici y el carril de circulación motorizado, con cierto margen de seguridad para posibles caídas o adelantamientos sin ocupar el carril motorizado.

- Carriles amplios que permitan cruces y adelantamientos sin angustias.

- Segregación física del carril bici con el de la circulación motorizada mediante elementos separadores.

- Intersecciones seguras, especialmente en rotondas.

- Señales claras y anticipadas.

- Sistema de semáforos para las bicicletas.

- Iluminación adecuada.

- Pavimento en buen estado.

- Aparcamientos estratégicamente ubicados, visibles, seguros y fáciles.

\section{Separación del carril bici}

Es necesaria la separación del carril bici del carril de circulación motorizado, dando cierto margen de seguridad para eventuales caídas o adelantamientos sin ocupar el carril motorizado. Esta separación aporta mayor seguridad, dado que se evitarán situaciones de vulnerabilidad de las personas ciclistas ocupando el carril para vehículos motorizados. Pero también aporta sensación de seguridad, ya que una mayor distancia de los vehículos a motor permite el uso de la infraestructura ciclista con mayor tranquilidad. Esta sensación de seguridad está estrechamente vinculada con la aversión a la temeridad que presentan en mayor medida las mujeres. Por tanto, los carriles bici con la separación necesaria atraerán a un mayor número de mujeres ciclistas. 
Debe garantizarse esta separación durante todo el recorrido del carril bici, con una separación mínima de 0,5 metros, preferiblemente de 1 metro, en paralelo al trazado de la vía.

\section{Carriles amplios}

Los carriles deben ser suficientemente amplios para garantizar su uso de forma cómoda, incluyendo el uso por parte de personas usuarias con elementos anexos a su bici, como pueden ser los remolques. La amplitud del carril debe permitir los adelantamientos sin angustias, con espacio suficiente como para que circulen como mínimo dos bicicletas. Garantizando que hay suficiente espacio en espacios como los cruces, y especialmente en los carriles bici compartidos en ambas direcciones.

Del mismo modo de la separación del carril bici, podemos observar un relación directa entre la amplitud del carril y la sensación de seguridad que este aporta a las personas usuarias.

Los carriles deben ser de como mínimo 2,5 metros de ancho en carriles bidireccionales exclusivos, y de mínimo 3 metros (deseable de 3,4 metros) en carriles compartidos con las y los peatones. Debe garantizarse que las aceras adyacentes a estos carriles son de como mínimo 3 metros, las cunetas de 1,5 metros hasta el elemento separador con el carril, franjas de seguridad de hasta 0,5 metros, y contar con parterres $\mathrm{u}$ otros elementos vegetales adyacentes a los carriles.

Estas distancias mínimas requieren en la mayoría de los casos que los carriles bici de sitúen en calles amplias, hecho que conecta con el principio de concurrencia de personas para oír y ser oído/a, dado que las calles anchas acostumbran a ser más concurridas.

\section{Segregación física del carril bici}

La instalación de elementos físicos que permitan segregar físicamente los carriles bici y motorizado garantizan la no invasión de los vehículos motorizados al carril bici, fortaleciendo la seguridad y la sensación de seguridad para las personas usuarias del carril bici.

Estos elementos físicos de separación pueden ser aceras, elementos de mobiliario urbano como jardineras, separadores de caucho, hormigón o barreras de seguridad como barandillas, pilonas flexibles, barreras metálicas bionda u otros elementos de protección.

\section{Intersecciones seguras}

Las intersecciones son uno de los puntos más críticos en cuanto a seguridad vial, tanto para las bicicletas como en términos generales para otros modos de transporte. Las personas usuarias de transporte activo como son las y los peatones y ciclistas son las que presentan mayor vulnerabilidad cuando se encuentran en una intersección. Para garantizar su seguridad es necesario poner la garantía de seguridad en el centro del diseño, condicionando al resto de la red de transporte.

Los elementos que nos permitirán integrar la seguridad en las intersecciones son elementos de seguridad vial como:

- Señales horizontales y verticales, como líneas de aviso de detención, ceda el paso, símbolos de bicicleta para distinguir los itinerarios, flechas de dirección en cada uno de los sentidos, distin- 
ción por color del tramo de carril bici. Este elemento concreto tiene una gran relación con el principio de señalización, dotando a las personas usuarias de las infraestructuras ciclistas de suficiente información para saber en qué carril y zona de la infraestructura deben situarse para garantizar la máxima seguridad durante su circulación.

- Paso elevado de vías peatonales y ciclistas, especialmente en intersecciones con vías de tren.

- Semáforos en las intersecciones, adaptando su configuración según la cantidad de tráfico.

- Iluminación enfatizada en las intersecciones.

\section{Iluminación adecuada}

La iluminación es un componente clave en la garantía de seguridad y sensación de esta. Tanto por motivos de seguridad vial por la correcta visibilidad de la circulación, como por motivos de seguridad personal. Una iluminación correcta aportará visibilidad de posibles agresores por parte de las personas usuarias; así como de la agresión misma en caso de cometerse. Permitiendo recibir socorro por parte de otras personas que se encuentren cercanas al lugar, vinculado al principio de acceso a la ayuda.

Este elemento está estrechamente ligado, además de al principio de acceso a la ayuda, con el principio de visibilidad. Una buena iluminación debe ir acompañada de un diseño del espacio que amplíe la visibilidad, evitando rincones, áreas escondidas o árboles que entorpezcan los focos de luz (Col-lectiu Punt 6, 2011).

Es necesario prestar especial atención a estos elementos en los casos de infraestructuras de transporte muy antiguas o en zonas de difícil acceso. Algunos organismos realizan auditorías de puntos negros durante las cuales se busca identificar aquellas zonas en las que las personas usuarias se pueden sentir inseguras. En algunas ocasiones no es suficiente con mejorar la iluminación en estos puntos, y en cambio es necesario ampliar las propuestas con soluciones más creativas e innovadoras, como pueden ser espejos o cambios en los materiales de las infraestructuras que permitan la visibilidad desde zonas cercanas.

\section{Pavimento en buen estado}

La calidad del pavimento y la pintura utilizada afecta directamente a la seguridad y sensación de esta, especialmente en las ocasiones de conducción difícil como puede ser la humedad en el suelo, hielo o nieve. Siguiendo el principio de mantenimiento de los lugares, es importante garantizar el mantenimiento del pavimento, resolviendo los problemas derivados de las raíces o charcos que puedan generar caídas o salidas de la vía.

\section{Aparcamientos}

Los aparcamientos de bicicletas deben situarse en lugares que generen confianza a las usuarias, evitando rincones oscuros y lugares traseros de edificios o alejados de las entradas o el tránsito. Vinculado al principio de planificación, aportando calidad de elemento acogedor a los aparcamientos al situarlos en lugares concurridos. 


\section{Conciliación}

Una de las partes más importantes del diseño de una infraestructura ciclista es la planificación de su recorrido. Cuando pensamos en el itinerario que debe realizar un carril bici, necesitamos pensar en los lugares a los que la población tiene necesidad de acceder, desde una perspectiva de movilidad y transporte, no de ocio (a no ser que se trate de infraestructuras ciclistas destinadas al ocio, como pueden ser las rutas ciclistas en ambientes naturales).

Así, un carril bici debe conectar los lugares a los que debe acceder la ciudadanía de forma cotidiana. Tradicionalmente, estos lugares son los centros laborales y las zonas residenciales de la ciudad. Como hemos comentado al inicio, incorporando la perspectiva de género, además de los centros laborales incluimos como actividades cotidianas vinculadas las tareas de cuidados. Es decir, aquellas vinculadas a la logística familiar. Estas tareas pueden ser las gestiones personales, las compras habituales y el cuidado de infantes y personas mayores. Por tanto, las infraestructuras ciclistas deberán permitir acceder a zonas de la ciudad donde desarrollar estas actividades. Convirtiendo las infraestructuras como los carriles bici en infraestructuras útiles para la movilidad activa de la ciudadanía al cargo de estas tareas, y por tanto de una mayor parte de la población en general. Bajo esta premisa, debemos tener en cuenta los siguientes elementos:

- Conectar centralidades como zonas de equipamientos vinculados a la actividad socio-económica de la ciudad.

- Conectar centros escolares y centros de cuidado para facilitar que infantes y otras personas dependientes puedan ser acompañados.

- Conectar zonas comerciales, integrando lugares donde realizar gestiones personales y compras cotidianas a la ruta diseñada.

- Conectar zonas residenciales y espacios urbanos.

- Conectar medios de transporte público.

- Instalar y facilitar zonas de aparcamiento cerca de estas zonas, para facilitar estas gestiones.

La mejora en las conexiones de estos espacios facilitará la vida cotidiana de muchas mujeres, facilitando el uso de la bicicleta durante las gestiones personales y las compras cotidianas. Pero también pueden suponer un incentivo para muchas personas para incorporar alguna de estas tareas en su vida cotidiana.

Una de las técnicas utilizadas para decidir qué zonas de la ciudad deben conectarse y establecer el itinerario de los carriles e infraestructuras ciclistas, es el análisis a partir del uso del Sistema de Información Geográfica (SIG). A partir de esta tecnología podemos clasificar las zonas de la ciudad según su funcionalidad, distinguir puntos de interés y situar el recorrido de los carriles bici y los aparcamientos correspondientes. En la Figura 2 podemos observar un ejemplo de mapa con clasificación de zonas, utilizado en el proyecto de pacificación de la Meridiana.

Para mayor detalle sobre la planificación urbanística usando el Sistema de Información Geográfica se recomienda consultar la bibliografía especializada de Angel Pueyo (1991) y Greg Rybarczyk y Wu Changshan (2010), por citar algunos ejemplos. 


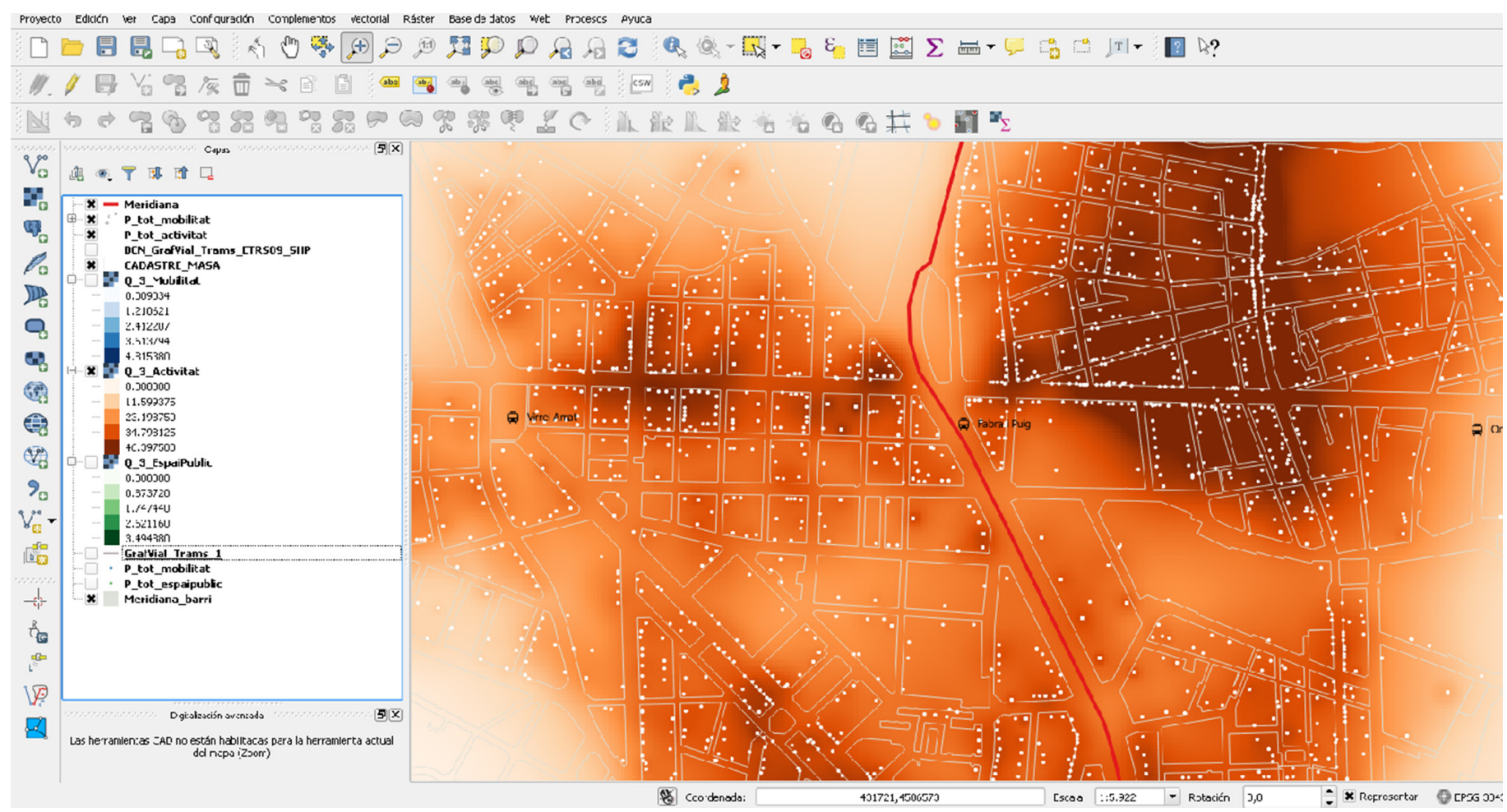

\section{Mirada integradora y participativa}

Cuando buscamos ampliar la proporción de la ciudadanía que usa la bicicleta como modo habitual de transporte, pretendemos incluir realidades sociales diversas. De este modo, las soluciones aspiran a poder incluir a toda la ciudadanía independientemente de su género, edad, procedencia cultural, bagaje religioso, capacidades y nivel socio-económico.

Es importante analizar las limitaciones que algunas de estas personas pueden tener que afrontar cuando quieren hacer uso de, en nuestro caso, la infraestructura ciclista y el uso de la bicicleta como medio de transporte. A modo de ejemplo, existen experiencias muy positivas en las que algunas ciudades han organizado cursos para aprender a ir en bicicleta dirigidos a personas adultas, como por ejemplo mujeres migrantes. $\mathrm{O}$ las experiencias de algunas ciudades en la promoción de los caminos escolares en bici, que permiten a familias y personas jóvenes acceder al centro de estudios en bicicleta.

Para poder garantizar que las realidades de cuantos más grupos sociales posibles se tienen en cuenta durante los procesos de diseño, es necesario incluir elementos inclusivos como los que estamos comentando. Además, es imprescindible incluir procesos de participación que permitan recoger demandas y necesidades diversas. Estos procesos participativos deben realizarse de la forma más inclusiva posible, garantizando la participación de la mayor parte de la ciudadanía posible, aplicando metodologías de participación que puedan atraer personas de diferentes perfiles. Por ejemplo, planificar una combinación de técnicas participativas presenciales y telemáticas, contando con la participación de entidades, con procesos participativos como entrevistas, acciones en el espacio público, talleres de diagnóstico, talleres de propuestas y encuestas. La combinación de estas metodologías de participación permitirá diversificar la población participante. La recogida de estos datos y propuestas debe recogerse de forma desagregada por género y grupos de edad.
Figura 2. Mapa de clasificación de zonas visualizado en el programa informático QGIS. Fuente: Elaboración propia en el marco del proyecto de pacificación de la Meridiana. 
Figura 3. Cartel promocional SQV SMART PAE. Fuente: (Federació de Municipis de Catalunya y Fundació Carles Pi i Sunyer, 20171.
Estas sesiones participativas pueden aportar información clave durante diversas fases del ciclo de política pública, especialmente durante el diagnóstico, el diseño y la evaluación. Los datos recogidos durante las sesiones participativas pueden ser incorporados al análisis por SIG, clasificando las necesidades de los diferentes grupos poblacionales. Así, pueden identificarse diferentes carencias durante la fase de diagnóstico, señalar algunas necesidades durante la fase de diseño y recoger la experiencia diversa del conjunto de la ciudadanía durante la fase de evaluación. Permitiendo un uso inclusivo de la infraestructura ciclista durante su fase de implementación. En la Figura 3 podemos observar diversos mapas de clasificación de necesidades, según el grupo poblacional. El uso de diversos mapas nos permite observar la coincidencias y discrepancias entre grupos poblacionales.

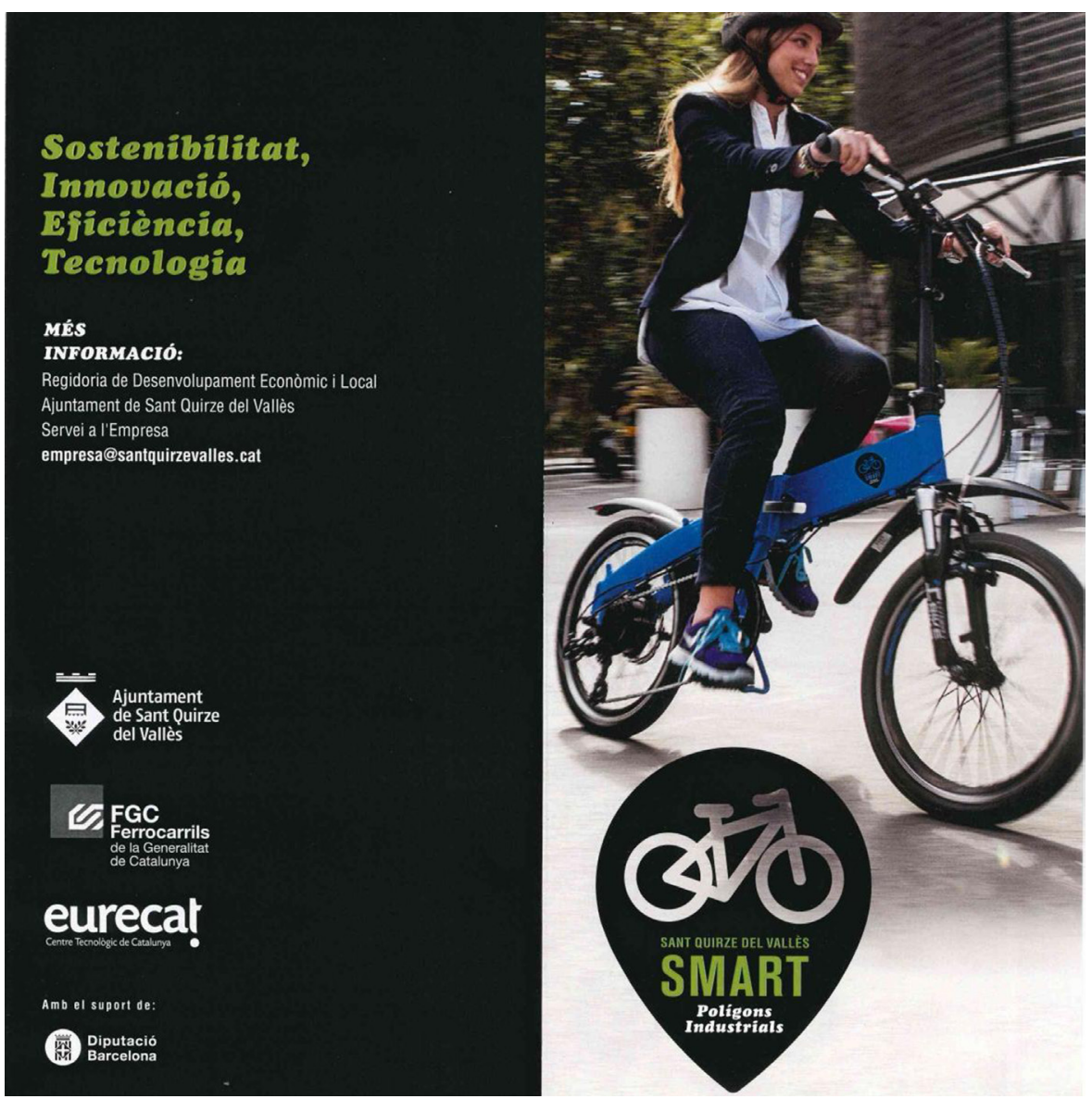

\section{Visibilidad y promoción}

Un elemento común de todas las políticas de género es incorporar la perspectiva de género en los elementos comunicativos vinculados al área de intervención. En el caso del uso de la bicicleta, es necesario tener en cuenta la representación de mujeres ciclistas en las actuaciones promocionales, presentando a las mujeres como sujetos activos de la acción de desplazarse en bicicleta.

A modo de ejemplo, durante la experiencia SQV SMART PAE que impulsó el Ayuntamiento de Sant Quirze del Vallès conjunto con Ferrocarriles de la Generalitat de Catalunya y el Centro Tecnológico Eurecat, para mejorar el acceso a los polígonos de actividad económica o PAE, se optó por la representación de mujeres en el uso de las infraestructuras implementadas, como puede verse en la Figura 4. 

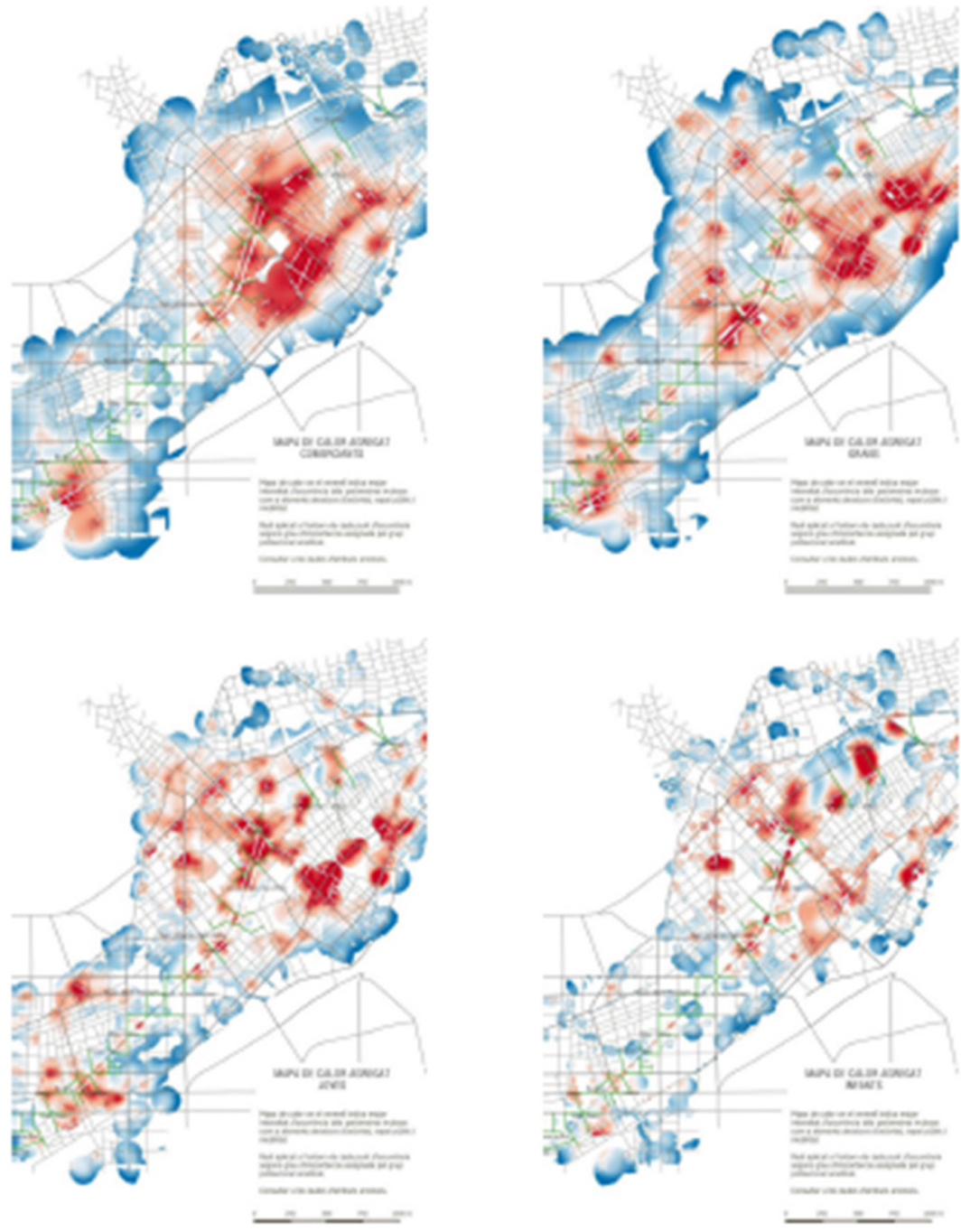

4

Pero también debe tenerse en cuenta en los elementos viarios, evitando la invisibilidad de las mujeres como ciclistas e intentando ampliar la representación de las personas usuarias de forma no discriminatoria, como pueden ser las figuras utilizadas en la señalización horizontal o los semáforos. Para una reflexión general y herramientas para aplicar una señalización urbana igualitaria en nuestras ciudades y municipios, se recomienda el trabajo de la Federación Española de Municipios y Provincias (2009).

\section{Conclusiones}

A lo largo de nuestras reflexiones hemos intentado demostrar cómo la incorporación de la perspectiva de género en el diseño de nuestras infraestructuras ciclistas requiere algo más que incorporar algunos pequeños elementos o implementar una serie de medidas prefabricadas. Para realizar el ejercicio de inclusión con potencial verdaderamente transformador será necesario repensar el modo en el que afrontamos el proyecto desde el inicio del proceso de diseño, incorporando metodologías participativas. Además, será necesario tener en cuenta algunos aspectos que hasta el momento habían estado olvidados, como pueden ser las tareas de cuidados, la seguridad de los modos de trans-
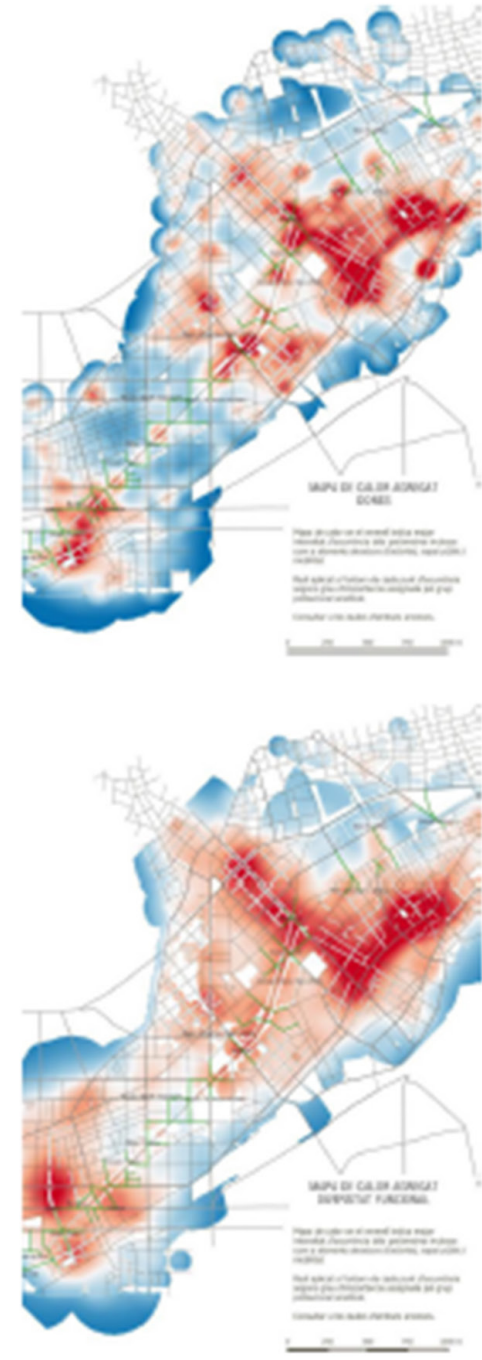

Figura 4. Mapa de necesidades según grupo poblacional. Fuente: Elaboración propia. 
porte activos, o la centralidad de las infraestructuras de transporte, por poner alguno de los ejemplos que hemos ido desarrollando.

Como hemos visto, una mejora en las condiciones de seguridad, calidad y comodidad de las infraestructuras ciclistas potenciará su uso por parte de mujeres, pero también por parte de otros colectivos como las personas con diversidad funcional, las personas mayores y las y los infantes. Unas infraestructuras ciclistas más amables suponen una acogida de más mujeres usuarias, pero también suponen un impacto positivo en el uso de estas infraestructuras para el conjunto de la ciudadanía. Maximizando el potencial sostenible de las infraestructuras de transporte impulsadas en los entornos de nuestras ciudades.

Para ello, es recomendable analizar los procesos de política pública a partir de los elementos destacados en la siguiente check-list.

\begin{tabular}{|c|c|c|}
\hline PARTICIPACIÓN & Sesiones participativas & $\begin{array}{l}\text { Inclusión de sesiones participativas durante diferentes fases del pro- } \\
\text { ceso de política pública }\end{array}$ \\
\hline \multirow{11}{*}{ SEGURIDAD } & \multirow{2}{*}{ Carril separado } & Carril separado más de 1 metro \\
\hline & & Carril separado más de 50 centímetros \\
\hline & \multirow{2}{*}{ Carril segregado } & Carril segregado con elementos físicos continuos \\
\hline & & Carril segregado con elementos físicos intermitentes \\
\hline & \multirow{3}{*}{ Intersecciones } & Intersecciones semaforizadas para bicicletas \\
\hline & & Intersecciones señalizadas para bicicletas \\
\hline & & Rotondas adaptadas a bicicletas \\
\hline & Anchura carril & $\begin{array}{l}\text { Permite el cruce con otra bicicleta o el adelantamiento de forma } \\
\text { holgada. Teniendo en cuenta los carros de carga y trasportines. }\end{array}$ \\
\hline & Pavimento & Asfaltado, regular y antideslizante \\
\hline & Iluminación & $\begin{array}{l}\text { Adecuada a lo largo de todo el recorrido y especialmente en las in- } \\
\text { tersecciones, en los aparcamientos de bicicletas y en zonas solitarias }\end{array}$ \\
\hline & Aparcamientos & $\begin{array}{l}\text { Estratégicamente colocados (en equipamientos, centros socioeconó- } \\
\text { micos...), visibles, iluminados y suficientes }\end{array}$ \\
\hline \multirow{3}{*}{ VISIBILIDAD } & Plan de comunicación & Diseñar el plan con perspectiva de género \\
\hline & \multirow[t]{2}{*}{ Promoción } & $\begin{array}{l}\text { Establecer targets en función del género y las diferentes realidades } \\
\text { sociales }\end{array}$ \\
\hline & & Utilizar imágenes de mujeres de diferentes realidades sociales \\
\hline \multirow[t]{2}{*}{ CONCILIACIÓN } & \multirow[t]{2}{*}{ Diseño del trazado } & $\begin{array}{l}\text { Diseñar el trazado para garantizar su funcionalidad durante la logís- } \\
\text { tica diaria de trabajo, compras u otras gestiones }\end{array}$ \\
\hline & & Pasar por escuelas, facilitando el acceso y aparcamientos seguros \\
\hline \multirow{3}{*}{ INCLUSIÓN } & Jóvenes en bicicleta & $\begin{array}{l}\text { Fomentar la movilidad en bicicleta a los centros de estudio, garanti- } \\
\text { zando el acceso y aparcamiento seguros }\end{array}$ \\
\hline & Escuela de la bicicleta & $\begin{array}{l}\text { Escuela de adultos para aprender a ir en bicicleta (especialmente } \\
\text { para grupos de mujeres migrantes) }\end{array}$ \\
\hline & Gente mayor en bicicleta & $\begin{array}{l}\text { Integrar los casales de gente mayor, centros de salud, farmacias y } \\
\text { otros servicios en el trazado del carril bici, para fomentar el uso de } \\
\text { la bicicleta de esta población }\end{array}$ \\
\hline
\end{tabular}


Este check-list pretende ser una herramienta de trabajo que nos facilite la tarea de incorporar la perspectiva de género durante todo el proceso de implementación de infraestructuras ciclistas en nuestras ciudades. Con la intención de observar qué elementos han estado abordados y cuáles quedan por afrontar.

Es importante señalar el hecho de que la incorporación de la perspectiva de género en cualquier tipo de política pública es un proceso que requiere de compromiso político y aprendizaje por parte de todas las partes implicadas. Los objetivos dependerán del organismo en cuestión y pueden modificarse a lo largo del tiempo. Por ello será importante comprender que se trata de un proceso de constante aprendizaje con interminables posibilidades. 


\section{Referencias bibliográficas}

Alfama i Guillén, Eva (2012). Les polítiques municipals de gènere. Barcelona: ICPS (Materials CiP: Articles Feministes, 12).

Alonso Álvarez, Alba (2015). El mainstreaming de género en España. Hacia un compromiso transversal con la igualdad. Valencia: Tirant lo Blanch.

Autoritat del Transport Metropolità - ATM (2018). Enquesta de mobilitat en dia feiner 2018 (EMEF 2018). Recuperado el 9 de abril de 2020 de: https:/ / www. atm.cat/web/ca/observatori/enquestes-de-mobilitat.php.

Asian Development Bank - ADB (2013). Gender Tool kit: Transport. Maximizing the Benefits of Improved Mobility for All. Avenue, Mandaluyong: ADB. Recuperado el 14 de abril de 2020 de: https:/ / www.adb.org/ sites/default/files/institutional-document/33901/ files/gender-tool-kit-transport.pdf.

Beall, Jo (1996). Participation in the City: Where Do Women Fit In? Gender E Development, 4(1). DOI : https://doi.org/10.1080/741921946.

Bofill Levi, Anna (2005). Planejament urbanistic, espais urbans $i$ espais interiors des de la perspectiva de les dones. Barcelona: Institut Català de les Dones-Generalitat de Catalunya. Recuperado el 3 de agosto de 2020 de: bit.ly/2lglm0F.

Campos de Michelena, Pascuala (1996). Influencia de las ciudades en la vida de las mujeres. Mujeres y urbanismo: una recreación del espacio. Claves para pensar en la viudad y el urbanismo desde una perspectiva de género. Madrid: Ministerio de Asuntos Sociales, Instituto de la Mujer, FEMP.

Ciocoletto, Adriana (2014). Urbanismo para la vida cotidiana. Herramientas de análisis y evaluación urbana a escala de barrio desde la perspectiva de género (Tesis doctoral). Universitat Politècnica de Catalunya, Barcelona. Recuperado el 3 de agosto de 2020 de: bit. ly/2MDea9O.

Cioccoletto, Adriana; y Col-lectiu Punt 6 (2014). Espacios para la vida cotidiana. Auditoría de Calidad Urbana con perspectiva de Género. Barcelona: Editorial Comanegra. Recuperado el 10 de abril de 2020 de: http://www.punt6.org/wp-content/ uploads/2016/08/EspaciosParalaVidaCotidiana. pdf.

CIVITAS (2014). Smart choices for cities. Gender equality and mobility: mind the gap! Recuperado el 14 de abril de 2020, de: http:/ / civitas.eu/sites/default/files / civ_pol-an2_m_web.pdf.

Col-lectiu Punt 6 (2011). Construyendo entornos seguros desde la perspectiva de género. Barcelona: ICPS (Col-leccions CiP: Informes, 5). Recuperado el 17 de abril de 2020 de: https:/ / punt6.files.wordpress. com/2011/03/construyendoentornosseguros.pdf.
Col-lectiu Punt 6 (2019). Urbanismo feminista. Por una transformación radical de los espacios de vida. Barcelona: Virus Editorial, Barcelona.

Dirección General de Tráfico - DGT (2019). Barómetro de la bicicleta en España. Informe de resultados. Barcelona: GESOP. Recuperado el 9 de abril de 2020 de: https:/ / www.ciudadesporlabicicleta.org/wp-content/uploads /2019/12/RCxB-Bar\%C3\%B3metrode-la-Bicicleta-2019.pdf.

Directorate-General for Research and Innovation (2011). Toolkit Gender in EU-funded research. Brussels: European Commission. Recuperado el 14 de abril de 2020 de: https://op.europa.eu/en/publicationdetail/-/publication/c17a4eba-49ab-40f1-bb7b-bb6 faaf8dec8.

European Bank for Reconstruction and Development - EBRD (2011). Gender 1. Urban rehabilitation and transport projects. Guidance Note. Recuperado el 14 de abril de 2020 de: https:/ / www.ebrd.com/news / publications/guides/gender-1-urban-rehabilitationand-transport-projects.html.

European Institute for Gender Equality - EIGE (2019). Gender planning. Luxembourg: Publications Office of the European Union. Recuperado el 14 de abril de 2020 de: https:/ / eige.europa.eu/sites / default/files/mh0319273enn_002_0.pdf.

Federació de Municipis de Catalunya y Fundació Carles Pi i Sunyer (2017). Banc de bones pràctiques [Base de datos]. Recuperado el 9 de abril de 2020 de: http:/ / www.bbp.cat/ficha_completa_red.php?paso =compl\&ficha=1293\&con=fora\&su_idioma=2\&chi=t.

Federación Española de Municipios y Provincias FEMP (2019). Manual práctico para una señalización urbana igualitaria. Recuperado el 12 de abril de 2020 de: http:/ /femp.femp.es/files/566-187-archivo/Manual\%20pr\%C3\%A1citico\%20para\%20 una $\% 20$ se $\%$ C3\%B1alizaci\%C3\%B3n\%20igualitaria.pdf.

Grupo de especialistas en Mainstreaming EG-S-MS (1999). Mainstreaming de género. Marco conceptual, metodología y presentación de buenas prácticas. Madrid: Ministerio de Trabajo y Asuntos Sociales. Instituto de la Mujer.

Ilárraz, Imanol (2006). Movilidad sostenible y equidad de género. Zerbitzuan, 40, 61-66.

Judge, Ainsley Henry (2011). Designing More Inclusive Streets: the Bicycle, Gender and Infrastructure. Geography Honor Projects, Paper 29.

Miralles-Guasch, Carme (2010). Dones, mobilitat, temps $i$ ciutats. Barcelona: Institut Català de les Dones-Generalitat de Catalunya. Recuperado el 3 de agosto de 2020, de: bit.ly/2lpx6xW.

Pueyo Campos, Angel (1991). El sistema de informa- 
ción geografica: un instrumento para la planificación y gestión urbana. Geographicalia, 28, 175-192.

Rybarczyk, Greg; y Changshan, Wu (2010). Bicycle facility planning using GIS and multi-criteria decision analysis. Applied Geography, 30(2), 282:293. DOI: https://doi.org/10.1016/j.apgeog.2009.08.005.
Zucchini, Elena (2015). Género y transporte: análisis de la movilidad del cuidado como punto de partida para construir una base de conocimiento más amplia de los patrones de movilidad. El caso de Madrid. (Tesis doctoral). Universidad Politécnica de Madrid.

Obach Lapieza, Anna; y Ramos Sanz, María (2020). Elementos clave para la introducción de la perspectiva de género en las infraestructuras ciclistas. Hábitat y Sociedad, 13, 87-105.

<http://dx.doi.org/10.12795/HabitatySociedad.2020.i13.06> 


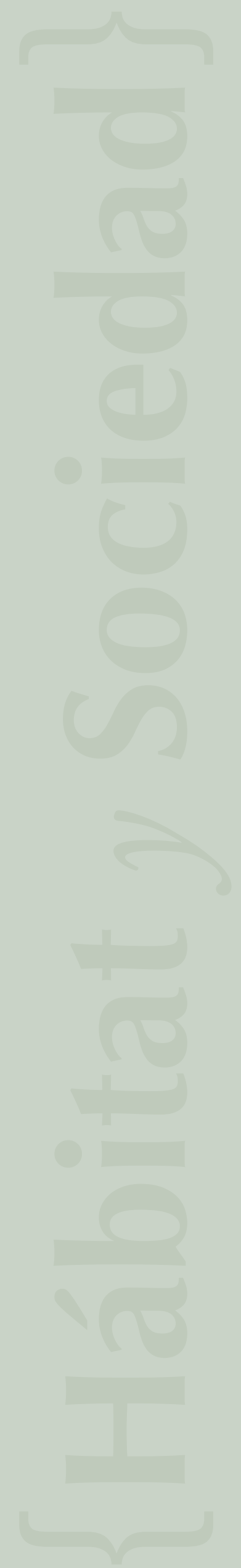

